\title{
Effects of X Capacitors on EMI Filter Effectiveness
}

\author{
Hung-I Hsieh, Student Member, IEEE, Jhong-Shu Li, and Dan Chen, Fellow, IEEE
}

\begin{abstract}
The suppression mechanism of the differential-mode noise of an $X$ capacitor in offline power supplies is, for the first time, attributed to two distinct concepts: 1) impedance mismatch (regarding a line impedance stabilization network or mains and the equivalent power supply noise source impedance) and 2) $C(d v / d t)$ noise current balancing (to suppress mix-mode noise). The effectiveness of $X$ capacitors is investigated with this theory, along with experimental supports. Understanding of the two aforementioned mechanisms gives better insight into filter effectiveness, which may lead to a more compact filter design.
\end{abstract}

Index Terms-Impedance mismatching, mix-mode (MM) electromagnetic interference (EMI) noise, noise current balancing.

\section{INTRODUCTION}

$\mathbf{E}$ LECTROMAGNETIC interference (EMI) filters have been widely used for years to solve conducted EMI problems for offline switching power supply applications. However, even up to date, some sort of "cut-and-try" effort has been necessary in normal design process for the filter. Progress has been made in the recent past in this regard, and new methodologies have been reported for the analysis and design of the EMI filters [1]-[4]. However, in the authors' opinion, there are two major problem areas that are still confounding to the designers. One is the proximity electromagnetic coupling problems of high frequency in filter components and the packaging environment [5]. The other area arises from the fact that there is mix-mode (MM) noise in addition to the differential-mode (DM) and commonmode (CM) noise conventionally associated with offline power converters [6]-[10]. Generally speaking, the former problem area occurs in the upper end, and the latter occurs in the lower end of the conducted EMI frequency spectrum.

This paper intends to address problems in the latter area. Discussions of the MM noise phenomenon have been reported in several recent papers [6]-[9]. However, the implications of the MM noise theory have not been fully exploited yet for filter design purposes and for resolution of some mysteries often cited by the designers. Investigation in this area revealed that some common questions confusing many designers in their efforts to minimize the filter size can be dealt with. Understanding of this intricate phenomenon can sometimes be very useful in solving their problems and may provide guidance for improving their designs.

Manuscript received August 18, 2006; revised August 15, 2007. This work was supported by The National Science Council of Taiwan, R.O.C. under Award NSC92 2218 E 002 051, Award NSC93 2218 E 002 143, and Award NSC94 2218 E 002084.

The authors are with the National Taiwan University, Taipei 106, Taiwan, R.O.C. (e-mail: d92921005@ntu.edu.tw).

Digital Object Identifier 10.1109/TIE.2007.896258

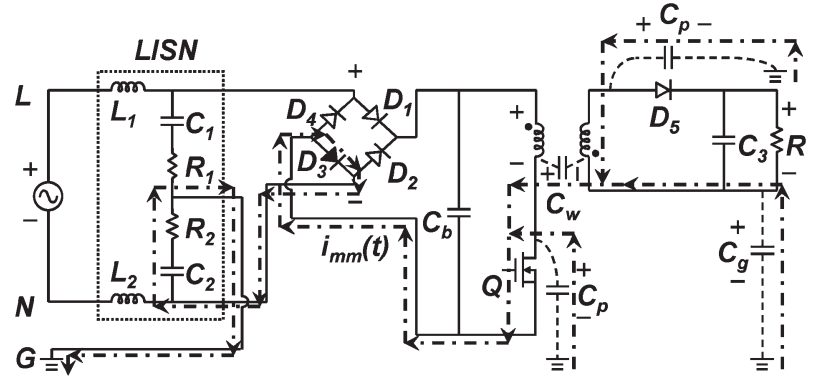

(a)

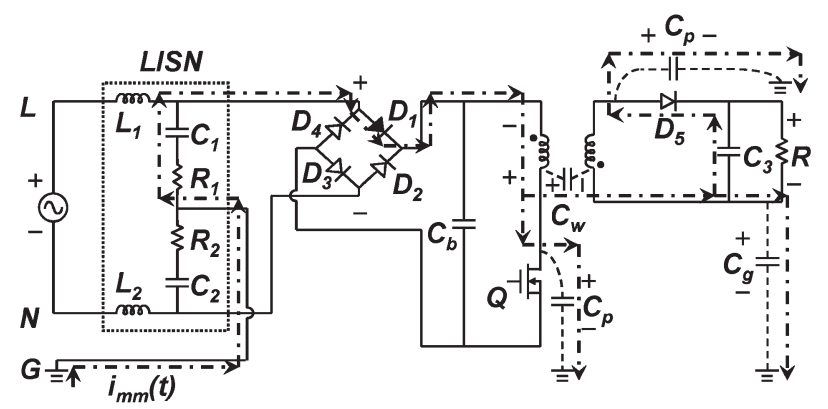

(b)

Fig. 1. Illustration of MM noise coupling. (a) Noise current flow during the "one-diode-conduction" period when MOSFET $Q$ is "on," diode $D_{5}$ is "off," and the parasitic capacitors are being discharged. (b) Noise current flow during the "one-diode-conduction" period when MOSFET $Q$ is "off," diode $D_{5}$ is "on," and the parasitic capacitors are being charged.

\section{INTRINSIC DM (IDM) NOISE AND MM NOISE}

Conducted EMI noise is normally classified into two modes: 1) $\mathrm{DM}$ noise and 2) $\mathrm{CM}$ noise. The recent addition, i.e., $\mathrm{MM}$ noise, accounts for some phenomenon that is unexplainable before. The MM noise is actually part of the DM noise, as seen from the line impedance stabilization network (LISN) side. It is the DM noise measured during the period when all the four bridge rectifying diodes of an offline power supply are thought to be off. During this period, contrary to conventional thinking, one of the diodes actually conducts high-frequency $C(d v / d t)$ current through parasitic capacitances in the circuit, as pointed out first in [7]. This current, if flowing through the two LISN resistors with imbalance, accounts for the DM noise measured during that period. Because the generating mechanism of this noise is similar to that of the CM noise but the suppression of the noise can be done with the DM filter, it is called MM noise. Fig. 1 shows the paths of the $C(d v / d t)$ current in an offline supply, illustrating the MM noise generation process. One can see that the current paths are different from those of when one pair of the rectifying diodes conducts; noise current also flows through the LISN resistors. This part of the DM noise, called the IDM noise, is conventionally known as the DM noise. To see 


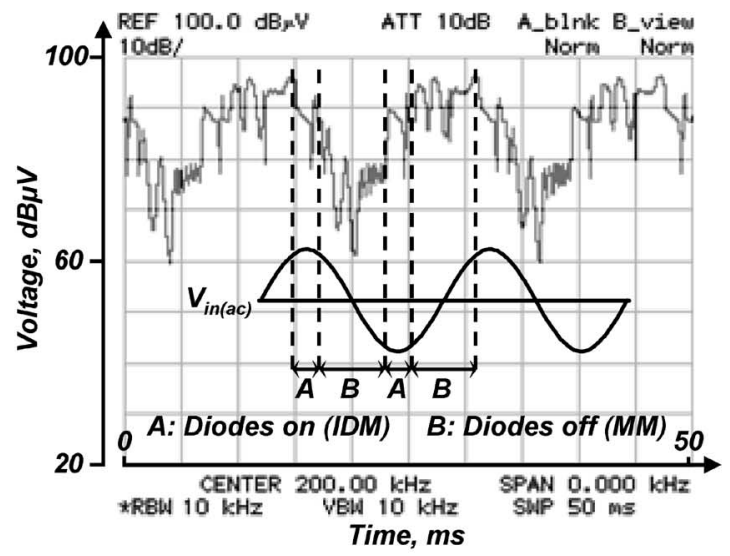

(a)

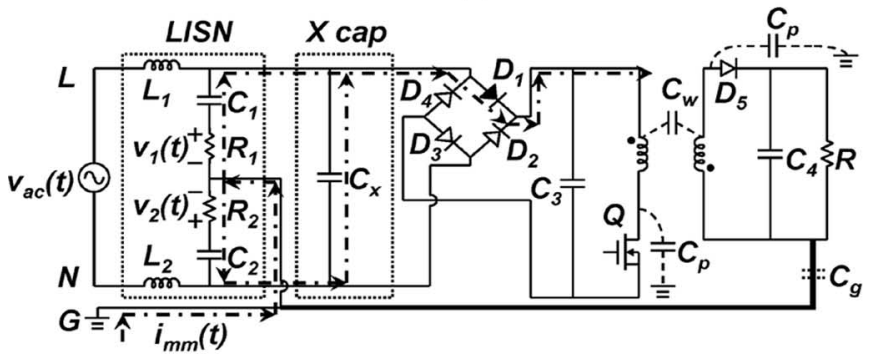

(b)

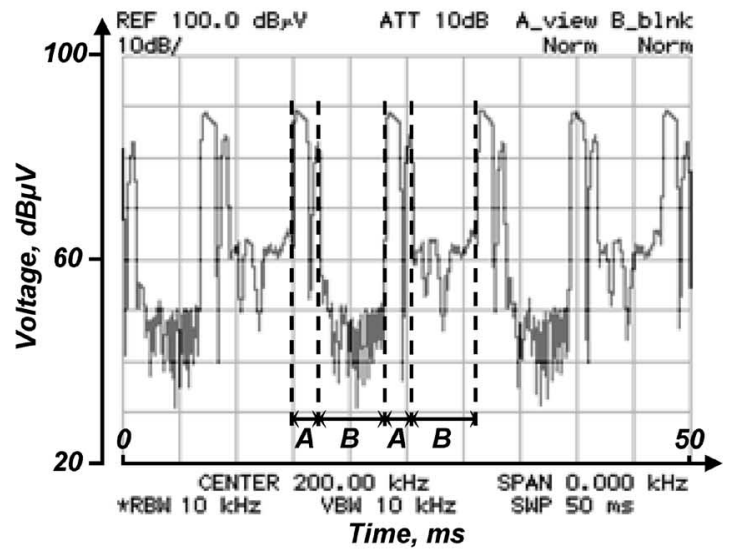

(c)

Fig. 2. Display of time-domain waveforms of the DM noise. Circuit operating conditions: $V_{\text {in }(\text { ac })}=110 \mathrm{~V}, P_{o}=40 \mathrm{~W}, V_{o}=12 \mathrm{~V}$, and the switching frequency is $50 \mathrm{kHz}$. (a) Zero-span mode (without $C_{x}$ ). (b) Balancing capacitor (with $C_{x}$ ). (c) Zero-span mode (with $C_{x}=0.68 \mu \mathrm{F}$ ); notice that the MM noise is much reduced but not the IDM noise.

the MM noise and the IDM noise on a spectrum analyzer, one can use the "zero span" mode of the analyzer, which displays the time-domain waveform of the noise spectrum at a selected frequency. Fig. 2(a) shows the time-domain waveform of the DM noise at the selected frequency of $200 \mathrm{kHz}$. In other words, DM noise at $200-\mathrm{kHz}$ frequency fluctuates with respect to time. In the figure, Period $\mathrm{A}$ is the diode conduction period, and Period B is the period when only one diode conducts. The noise measured is called IDM during Period A and MM during Period B. Fig. 1 shows the MM noise current flow when the input line voltage polarity is as shown. When the input voltage polarity changes, then $D_{2}$ or $D_{4}$ conducts. The larger level of the two determines the final DM EMI level at this frequency, if an EMI peak detector is used. If an average detector is used,

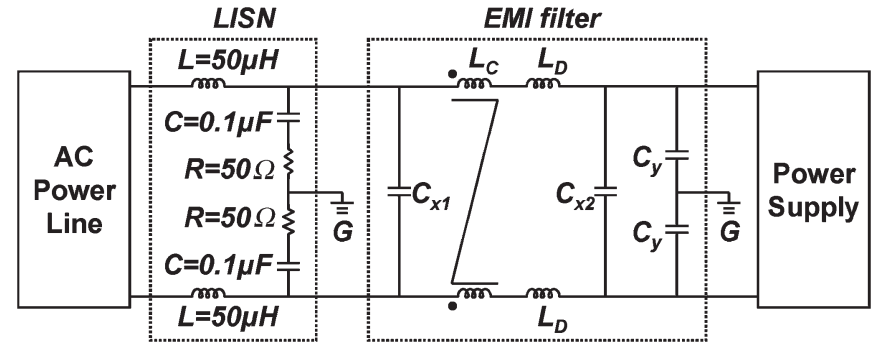

Fig. 3. Typical EMI filter topology.

then the final measurement levels are dependent on the magnitude proportion of the two levels and their relative duration [11]. When an $\mathrm{X}$ capacitor $C_{x}$ is added to the circuit, as illustrated in Fig. 2(b), then the noise current through the two LISN resistors is balanced, as indicated by the dotted lines. The MM noise is therefore much reduced for Period B, as shown in Fig. 2(c). Notice that very little noise attenuation occurs for IDM (Period A). Since conventional DM noise measurement takes the worse noise level (in this case, the IDM noise level during Period $\mathrm{A}$ is the worse), $C_{x}$ seems to be providing little EMI suppression. However, in the cases when MM dominates the overall DM noise, then $C_{x}$ would play a significant role.

Filtering of the complete DM noise, therefore, must be considered in two parts: one for IDM and the other for MM noise. Using the typical EMI filter topology shown in Fig. 3, the explanation of the DM filtering mechanism will be given here. The effectiveness of an X capacitor, according to conventional theory, depends on the degree of impedance mismatching [10] between the $\mathrm{X}$ capacitor and its paralleled element (either the power supply noise source impedance or the LISN impedance). This means that, if the impedance of the $\mathrm{X}$ capacitor is much smaller than the impedance of the paralleled element, then the attenuation would be effective. The elements in parallel with the two $X$ capacitors are the noise source impedance of the power supply (for $C_{x 2}$ ) and the LISN 100- $\Omega$ resistor (for $C_{x 1}$ ). So, for effective suppression of the IDM noise, the $\mathrm{X}$ capacitor used must be with low-enough impedance compared to the respective paralleled element. For effective suppression of the MM noise, however, the criterion is much different. The root cause of the MM noise is the unbalance flow of high-frequency $C(d v / d t)$ current through the two LISN 50- $\Omega$ resistors, as depicted in Fig. 1. When an $\mathrm{X}$ capacitor $C_{x}$ is used, however, the current that flows through the two LISN resistors becomes balanced if the impedance of $C_{x}$ is low compared to the rest of the path. Fig. 2(b) shows such a case. When the current path is balanced, the MM noise is much reduced. Therefore, the effectiveness of $C_{x}$ on MM noise suppression depends on the degree of LISN current balancing. To be effective in this regard, the impedance of the $\mathrm{X}$ capacitor $C_{x 1}$ or $C_{x 2}, Z_{C_{x 1}}$, and $Z_{C_{x 2}}$, respectively, must be insignificant compared to the impedance of the rest of the path. Referring to Fig. 3, the balancing conditions for the MM noise are expressed in (1) and (2). Notice that if (1) is satisfied, then the IDM noise also satisfies an impedance mismatch condition in (3). Notice that (2) is only valid for the MM noise and is not valid for the IDM noise. It is pointed out that, when (2) is satisfied, the two currents through the two windings of the CM choke are nearly 
balanced, not only in magnitude but also in phase. Therefore, the CM choke presents itself as inductance $L_{C}$ for each path.

\section{A. For $C_{x 1}$ (to Achieve Both MM Noise Current Balancing and Impedance Mismatch)}

$$
Z_{C_{x 1}}=\frac{1}{\omega \cdot C_{x 1}} \ll\left|50+\frac{1}{j \omega \cdot C}\right|
$$

where $C$ is the line frequency blocking capacitor of $0.1 \mu \mathrm{F}$ inside LISN. The impedance of $C$ at EMI-noise frequency is usually much less than $50 \Omega$.

\section{B. For $C_{x 2}$ (to Achieve MM Noise Current Balancing)}

$$
Z_{C_{x 2}}=\frac{1}{\omega \cdot C_{x 2}} \ll\left|50+\frac{1}{j \omega \cdot C}+j \omega \cdot\left(L_{C}+L_{D}\right)\right|
$$

where $L_{C}$ and $L_{D}$ are the inductance values of the CM choke and DM choke, respectively.

\section{For $C_{x 1}$ (to Achieve Impedance Mismatch)}

$$
Z_{C_{x 1}}=\frac{1}{\omega \cdot C_{x 1}} \ll\left|100+\frac{2}{j \omega \cdot C}\right| .
$$

An X capacitor is, therefore, serving both as an impedancemismatching capacitor and a balancing capacitor. The effectiveness of each function depends on different criteria. For example, $C_{x 1}$ is effective as an IDM filter only if its impedance is small compared to that of the LISN (100 $\Omega$ ). However, its effectiveness for suppressing MM noise lies on (1). Therefore, the same $C_{x 1}$ exerts different influences on the IDM part and the MM part of the DM noise.

CM choke $L_{C}$ plays no role in suppressing the conventional DM noise (i.e., the IDM noise called in this paper). This is because the IDM noise current flows into the dot in one winding and out of the dot in the other winding of $L_{C}$. However, for the MM noise, $L_{C}$ plays a role because the noise current flows through either only one leg of the CM choke or both legs but in the same direction. Either way, $L_{C}$ impedance is present in the path. This is clearly indicated in [7].

\section{AdDressing Some Common Questions}

There are questions often raised by practicing engineers about the elusiveness of filter issues. Some of the questions are related to the IDM/MM issue. It should be pointed out that one cannot see the full picture by just looking at the total EMI noise. Better knowledge of the "behind-the-scenes" CM, IDM, and MM noise would allow the designers to deal with the root causes, which may lead to a more compact filter design. Answers to some commonly raised questions will be given here. In all the experimental zero-span mode waveforms given, a selected frequency of 150 or 170 or $200 \mathrm{kHz}$ was used. For other frequencies, the theory is the same. It should be pointed out that, at high frequency, when the component resonance effect becomes pronounced, the values of capacitors and inductors are no longer independent of frequency. Under such a condition, (1) should be replaced by $Z_{C_{x 1}} \ll\left|50+Z_{C}\right|$, and (2) should be replaced by $Z_{C_{x 2}} \ll\left|50+Z_{C}+Z_{L_{C}}+Z_{L_{D}}\right|$, where the $Z$ 's represent the impedances of respective elements. The effect of frequency on this matter is dictated by (1) and (2). In addition, capacitors $C_{x}$ of 0.22 or $0.68 \mu \mathrm{F}$ were often used in the experiments. An X capacitor of $0.68 \mu \mathrm{F}$ satisfies (1), and an $\mathrm{X}$ capacitor of $0.22 \mu \mathrm{F}$ satisfies (2) at the selected frequencies.

\section{A. Relative Effectiveness of $C_{x 1}$ and $C_{x 2}$}

$C_{x 2}$ is, in general, not very effective in IDM noise suppression because of the low DM noise source impedance of an offline power supply [10]. However, it is effective for MM noise suppression, because it is relatively easier to satisfy (2). On the other hand, $C_{x 1}$ is relatively more effective for IDM suppression when connected in parallel to $100-\Omega$ total LISN resistance. It is relatively poorer for MM noise suppression, because (1) is more difficult to satisfy compared to (2) due to the presence of the inductors. The aforementioned assertion is supported by the experimental waveforms shown in Fig. 4. Fig. 4(a) shows the complete circuit diagram including the complete EMI filter. Fig. 4(b) shows the time-domain display of the DM noise at $170 \mathrm{kHz}$ for both cases: one without $C_{x 1}$ and $C_{x 2}$, and the other with $C_{x 1}=0$ but $C_{x 2}=0.22 \mu \mathrm{F}$. One can see that the suppression effect of $C_{x 2}$ is very evident for Period B (MM noise) but very little for Period A (IDM noise). However, when $C_{x 1}$ is added, the suppression for Period A is very evident, but that for Period B is slight, because the circuit has already been balanced before $C_{x 1}$ was added. Therefore, if a certain value of $C_{x 1}$ is necessary to keep the IDM noise under acceptable level, such capacitance value has to be large enough to satisfy (1). In that case, $C_{x 1}$ plays a major role in suppressing both the IDM noise and the MM noise. $C_{x 2}$ can be removed without much difference, as shown in Fig. 5. If the $C_{x 1}$ value that keeps IDM under acceptable level is not large enough to also meet (1), then there are two options: One option is to keep $C_{x 2}$ with a value that is barely large enough to meet (2). The other option is to eliminate $C_{x 2}$ but increase the $C_{x 1}$ value to the point where $C_{x 1}$ meets (1). It should be noted that the DM noise source impedance of an offline power supply is generally low because of a large bulk capacitor $\left(C_{3}\right)$ in this type of circuit. In current-fed power converters such as a powerfactor-correction boost configuration [12], the DM noise source impedance is high due to its inductive nature. In such a case, $C_{x 2}$ can be effective for IDM noise suppression.

Designers often ask the question, "If only one $\mathrm{X}$ capacitor is allowed, on which side should it be placed: the LISN side or the power-supply side?" The answer to this question depends on the IDM noise level before any X capacitor is used. For convenience of discussion, this level will be called the "pre-X cap" IDM level. If the pre-X cap IDM and MM level already exceeds the specification, then $C_{x 1}$ should be used, i.e., an X cap should be placed on the LISN side. The $C_{x 1}$ value would 


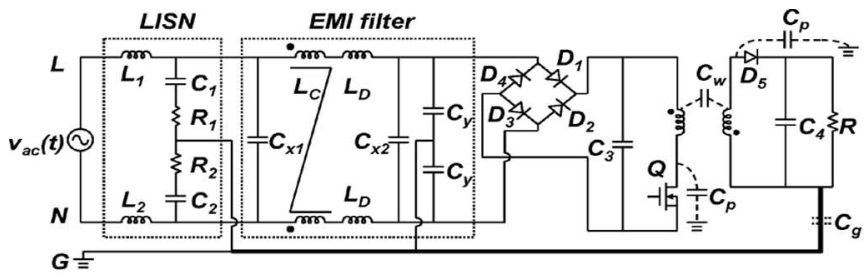

(a)

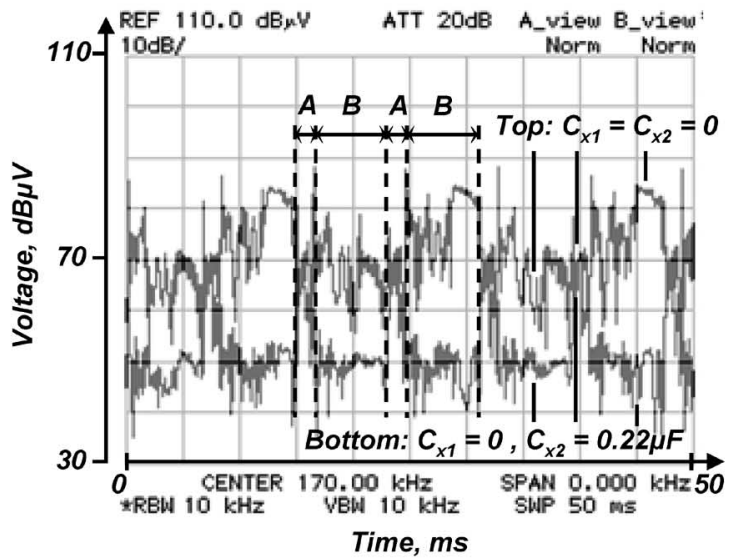

(b)

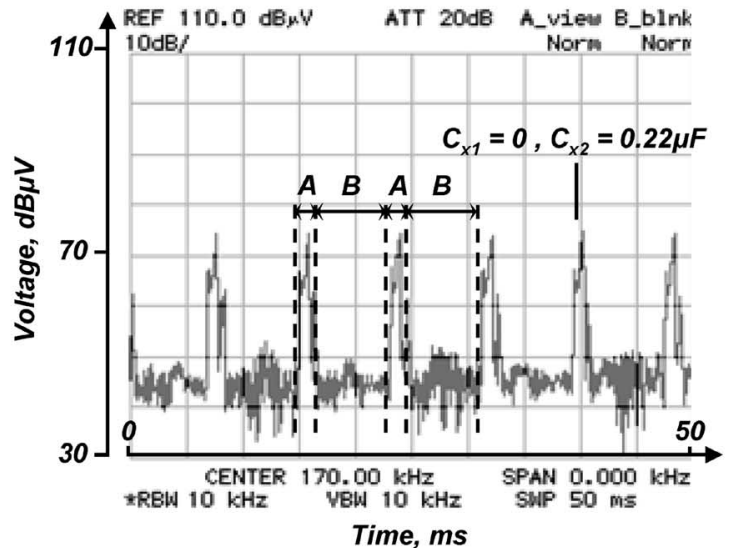

(c)

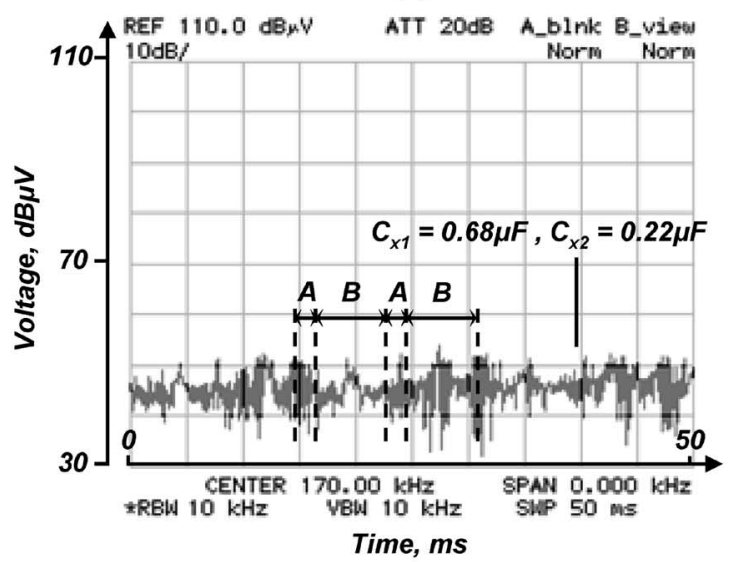

(d)

Fig. 4. Effectiveness of $C_{x 1}$ and $C_{x 2}$ under various conditions. (a) Complete diagram with the EMI filter $\left(L_{D}=100 \mu \mathrm{H}, L_{C}=8.5 \mathrm{mH}, C_{y}=3300 \mathrm{pF}\right)$. Circuit operating conditions: See Fig. 2 caption. (b) Zero-span mode (top: $C_{x 1}=C_{x 2}=0$; bottom: $C_{x 1}=0, C_{x 2}=0.22 \mu \mathrm{F}$ ). (c) Zero-span mode $\left(C_{x 1}=0, C_{x 2}=0.22 \mu \mathrm{F}\right)$. (d) Zero-span mode $\left(C_{x 1}=0.68 \mu \mathrm{F}, C_{x 2}=\right.$ $0.22 \mu \mathrm{F})$.

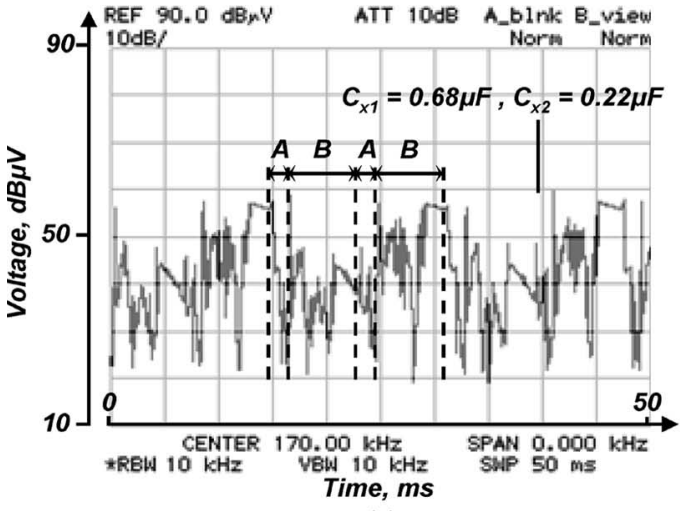

(a)

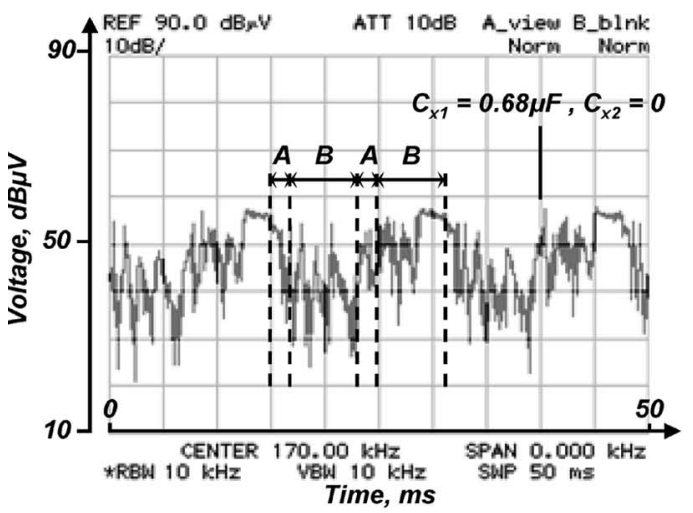

(b)

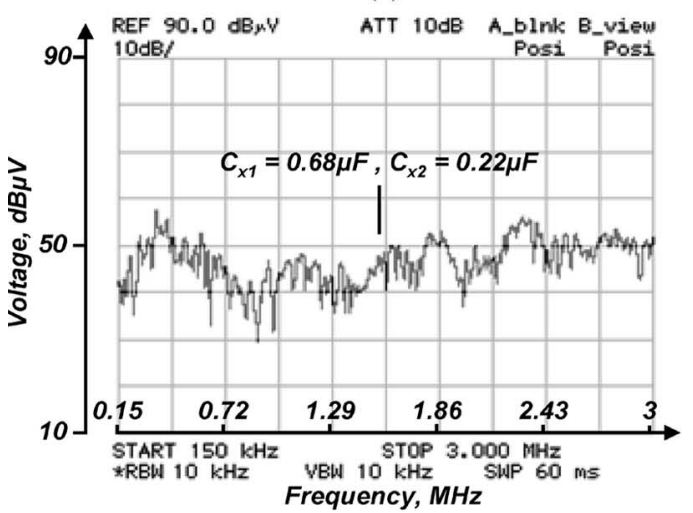

(c)

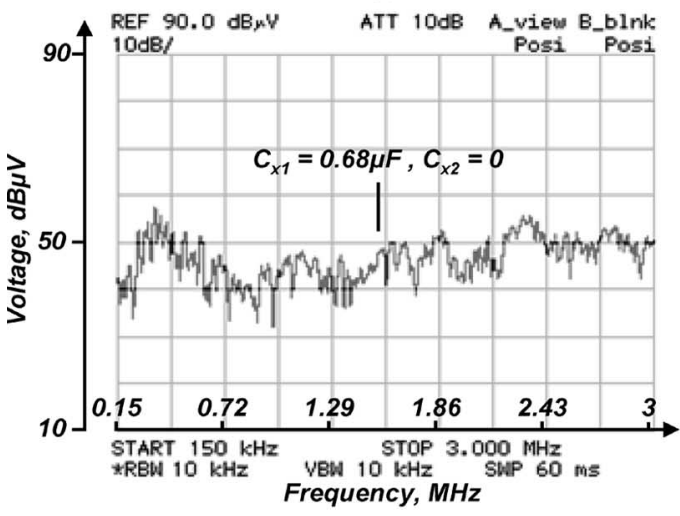

(d)

Fig. 5. $C_{x 2}$ be removed and DM noise without much difference. (a) Zerospan mode $\left(C_{x 1}=0.68 \mu \mathrm{F}, C_{x 2}=0.22 \mu \mathrm{F}\right)$. (b) Zero-span mode $\left(C_{x 1}=\right.$ $\left.0.68 \mu \mathrm{F}, C_{x 2}=0\right)$. (c) DM EMI noise for a range of frequency of $150 \mathrm{kHz}-$ $3 \mathrm{MHz}\left(C_{x 1}=0.68 \mu \mathrm{F}, C_{x 2}=0.22 \mu \mathrm{F}\right)$. (d) DM EMI noise for a range of frequency of $150 \mathrm{kHz}-3 \mathrm{MHz}\left(C_{x 1}=0.68 \mu \mathrm{F}, C_{x 2}=0\right)$. 


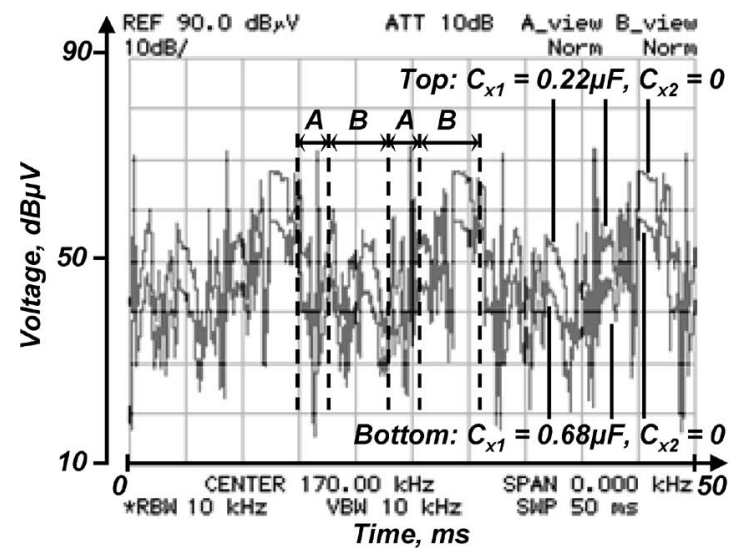

Fig. 6. Zero-span mode ( $C_{x 1}$ can be effective in suppressing the MM noise when $C_{x 2}$ is not present).

have to be enough for both suppressing IDM and meeting (1). Fig. 6 shows the waveform comparison when the $C_{x 1}$ value was increased from 0.22 to $0.68 \mu \mathrm{F}$, with $C_{x 2}=0$. It can be seen that $C_{x 1}$ is effective in suppressing both the IDM and MM noise levels when its value is increased. Notice that $C_{x 2}=0$ in the experiment leading to Fig. 6. When $C_{x 2}$ is absent, there is no effective way to suppress the MM noise, and the burden is shifted to $C_{x 1}$. As long as $C_{x 1}$ satisfies (1), then it is effective. However, this may lead to a much larger $C_{x 1}$ than the case of adding $C_{x 2}$. On the other hand, if the pre-X cap IDM noise level is below the specification, but the MM noise level is above it, then $C_{x 2}$, which is the $\mathrm{X}$ cap on the power-supply side, should be used. Fig. 4(b) shows the effectiveness of $C_{x 2}$ on the MM noise but not that on IDM. However, since IDM is not a concern in this case, it does not matter. One can see from preceding discussions why the filter issue is so elusive. The solution depends much on particular conditions and may not be generalized. Understanding of the issues allows sophisticated designers to have a better grip on this elusive subject. It should be pointed out that the one-cap solution may not be optimal, because the two-cap solution may end up with less total capacitance.

\section{B. Oversizing X Capacitance}

Sometimes, increasing the $\mathrm{X}$ cap value does not affect the total DM noise. The zero-span waveforms used in the discussion tell the insight of the nature of noise. The peak value of the waveform becomes the magnitude of the noise at that particular frequency in a normal frequency spectrum noise display. If the MM noise dominates and the $\mathrm{X}$ cap is primarily used for IDM suppression, then increasing $X$ capacitance would not affect the MM noise and therefore would not be noticeable from the frequency spectrum display. On the other hand, if the total DM noise is dominated by the IDM noise, then increasing $C_{x 2}$ may not be helpful to the total DM noise, because $C_{x 2}$ is, in general, not very effective for IDM suppression for voltage-fed converters. For current-fed converters, the situation is different. This fact has been pointed out in Section III-A. Fig. 7 illustrates this assertion. Increasing $C_{x 2}$ does not help the overall DM noise.

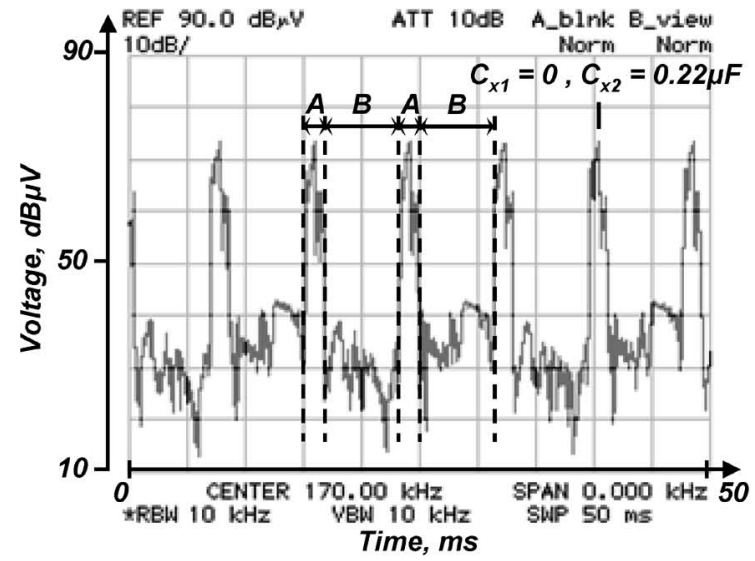

(a)

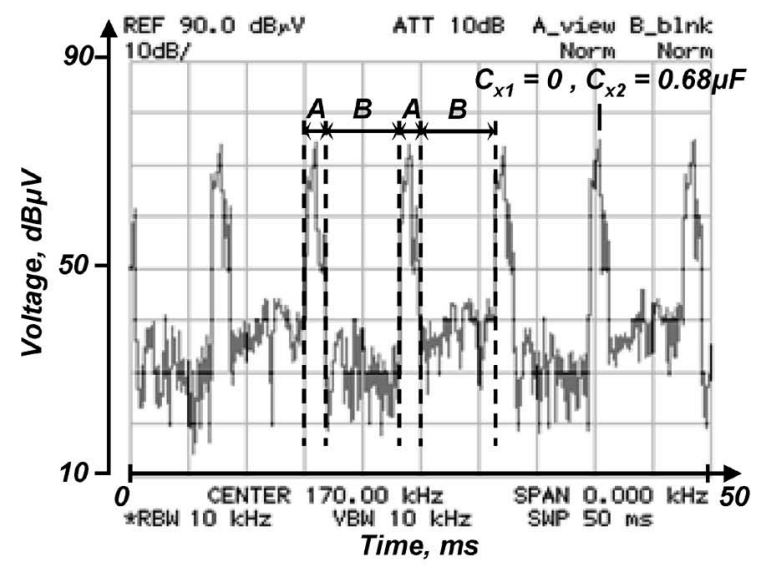

(b)

Fig. 7. Increasing $C_{x 2}$ does not help the overall DM noise. (a) Zero-span mode $\left(C_{x 1}=0, C_{x 2}=0.22 \mu \mathrm{F}\right)$. (b) Zero-span mode $\left(C_{x 1}=0, C_{x 2}=\right.$ $0.68 \mu \mathrm{F})$.

\section{Utilizing the Y Capacitors as a Balancing Capacitance}

Y capacitors alone can be a balancing cap as long as their impedance meets the balancing condition

$$
Z_{\frac{1}{2} C_{y}}=\frac{2}{\omega \cdot C_{y}} \ll\left|50+\frac{1}{j \omega \cdot C}+j \omega \cdot\left(L_{C}+L_{D}\right)\right| .
$$

Because of the small $C_{y}$ value normally used, the chance of meeting (4) is enhanced if there are chokes used in the filter. It is easier to meet (4) if the $\mathrm{Y}$ capacitors are placed on the powersupply side. Because of the relatively large CM noise source impedance associated with a power supply [10], it is also better to put the $\mathrm{Y}$ capacitors on the power-supply side for $\mathrm{CM}$ noise suppression.

\section{Effect of $C_{x}$ on CM Noise}

The fact that balancing provided by $C_{x}$ makes the two $C(d v / d t)$ currents equal implies that $\mathrm{CM}$ noise $V_{\mathrm{CM}}$, which is defined as the average of the two voltages across the two LISN $50-\Omega$ resistors that is $\left(V_{R 1}+V_{R 2}\right) / 2$, will be increased. The increase varies depending on how balanced the two paths after the addition of $C_{x}$ are. The largest increase is two times, which is 


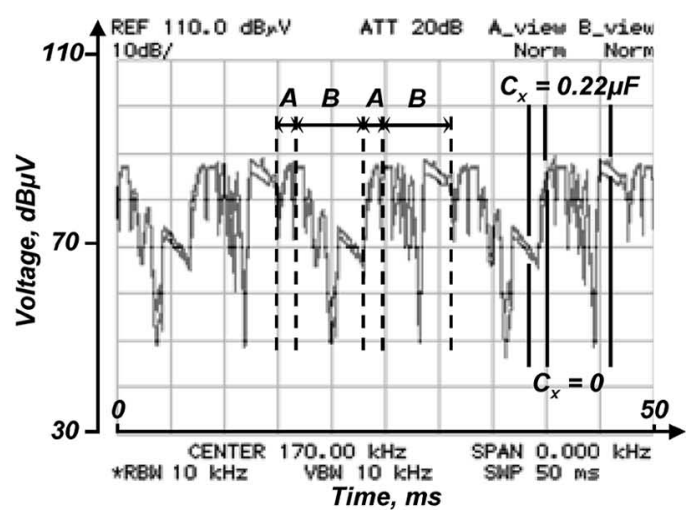

(a)

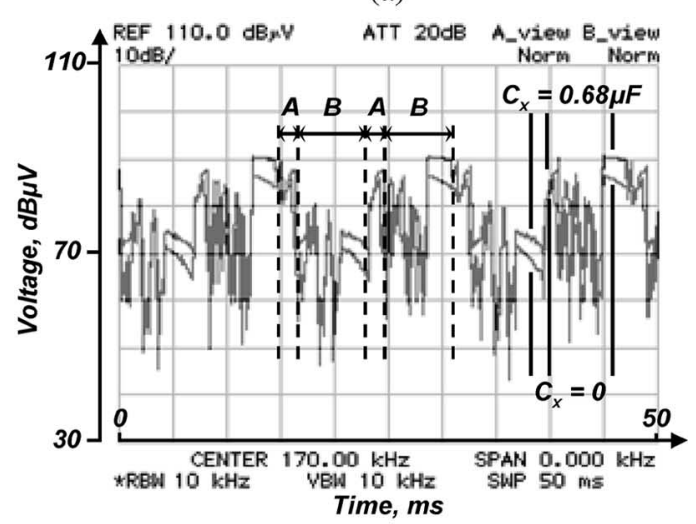

(b)

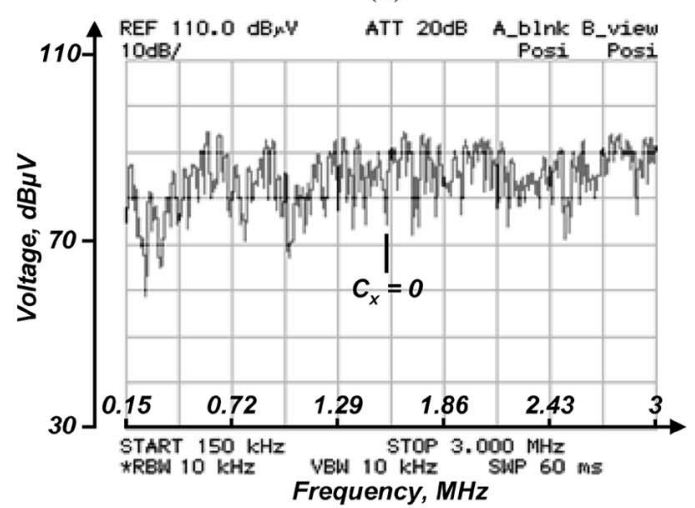

(c)

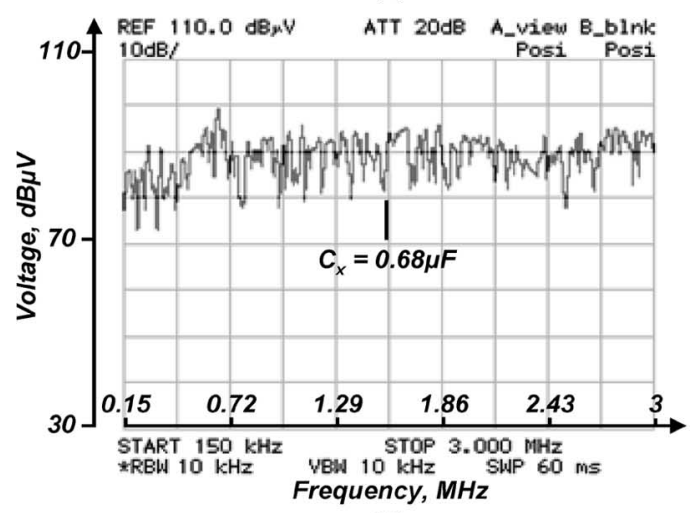

(d)

Fig. 8. Increase of the $\mathrm{CM}$ noise due to usage of a $C_{x}$. (a) Zero-span mode in the CM noise (top: $C_{x}=0.22 \mu \mathrm{F}$, bottom: $C_{x}=0$ ). (b) Zero-span mode in the CM noise (top: $C_{x}=0.68 \mu \mathrm{F}$, bottom: $C_{x}=0$ ). (c) CM EMI noise for a range of frequency of $150 \mathrm{kHz}-3 \mathrm{MHz}\left(C_{x}=0\right)$. (d) CM EMI noise for a range of frequency of $150 \mathrm{kHz}-3 \mathrm{MHz}\left(C_{x}=0.68 \mu \mathrm{F}\right)$.
$6 \mathrm{~dB}$, because the change from "no current flow" $\left(V_{\mathrm{CM}}=\right.$ $\left.\left(V_{R}+0\right) / 2\right)$ to "balanced current flow" $\left(V_{\mathrm{CM}}=\left(V_{R}+\right.\right.$ $\left.V_{R}\right) / 2$ ) in one LISN resistor means that the average across the two LISN $50-\Omega$ resistors increases by a factor of two. Fig. 8 shows the increase of the CM noise due to the existence of $C_{x}$.

\section{CONCLUSION}

Part of the DM noise is suppressed by impedance mismatching action, and the other part is suppressed by the balancing action of the $\mathrm{X}$ capacitors in EMI filters. The effectiveness of the two separate actions of an $\mathrm{X}$ capacitor varies with frequency, capacitance value, and filter topological arrangement. The phenomenon was explained, and mathematical conditions for effective filter attenuation were pointed out in this paper. Discussions of how one may exploit this new understanding to make a more compact filter were given. The optimal solution often depends on particular conditions and cannot be generalized. Understandings of the issues allow sophisticated designers to have a better grip on this elusive subject.

It should be pointed out that the MM noise is related to the use of an LISN, i.e., LISN allows a favorite noise current flow through earth ground. In the real case, where there is no LISN used, the actual noise emitted to the mains can differ from the noise measured, in which the use of LISN is mandatory. Whatever the difference, the measurement results under the LISN condition are what count.

\section{REFERENCES}

[1] J.-S. Lai, X. Huang, E. Pepa, S. Chen, and T. W. Nehl, "Inverter EMI modeling and simulation methodologies," IEEE Trans. Ind. Electron., vol. 53, no. 3, pp. 736-744, Jun. 2006.

[2] M. Jin and M. Weiming, "Power converter EMI analysis including IGBT nonlinear switching transient model," IEEE Trans. Ind. Electron., vol. 53, no. 5, pp. 1577-1583, Oct. 2006.

[3] W. Chen, X. Yang, and Z. Wang, "Analysis of insertion loss and impedance compatibility of hybrid EMI filter based on equivalent circuit model," IEEE Trans. Ind. Electron., vol. 54, no. 4, pp. 2057-2064, Aug. 2007.

[4] J. Meng, W. Ma, Q. Pan, Z. Zhao, and L. Zhang, "Noise source lumped circuit modeling and identification for power converters," IEEE Trans. Ind. Electron., vol. 53, no. 6, pp. 1853-1861, Dec. 2006.

[5] S. Wang, F. C. Lee, D. Y. Chen, and W. G. Odendaal, "Effects of parasitic parameters on EMI filter performance," IEEE Trans. Power Electron., vol. 19 , no. 3, pp. 869-877, May 2004.

[6] D. Zhang, D. Chen, and D. Sable, "Non-intrinsic differential mode noise caused by ground current in an off-line power supply," in Proc. IEEE Power Electron. Spec. Conf., May 1998, pp. 1131-1133.

[7] S. Qu and D. Chen, "Mixed-mode EMI noise and its implications to filter design in offline switching power supplies," IEEE Trans. Power Electron., vol. 17 , no. 4, pp. 502-507, Jul. 2002.

[8] M. Jin and M. Weiming, "A new technique for modeling and analysis of mixed-mode conducted EMI noise," IEEE Trans. Power Electron., vol. 19, no. 6, pp. 1679-1687, Nov. 2004.

[9] H.-I. Hsieh, D. Chen, and S. Qu, "A filter design procedure incorporating mixed-mode EMI noise for off-line switching power supplies," in Proc. IEEE Power Electron. Motion Control Conf., Aug. 2004, pp. 1527-1532.

[10] M. J. Nave, Power Line Filter Design for Switched-Mode Power Supply. New York: Van Nostrand Reinhold, 1991, ch. 2, sec. 2.3-2.6.

[11] M. L. Heldwein, T. Nussbaumer, and J. W. Kolar, "Differential mode EMC input filter design for three-phase AC-DC-AC sparse matrix PWM converters," in Proc. IEEE Power Electron. Spec. Conf., May 2004, pp. 284-291.

[12] S. Wang, F. C. Lee, and W. G. Odendaal, "Improving the performance of boost PFC EMI filters," in Proc. IEEE Appl. Power Electron. Conf. Expo., Feb. 2003, pp. 368-374. 


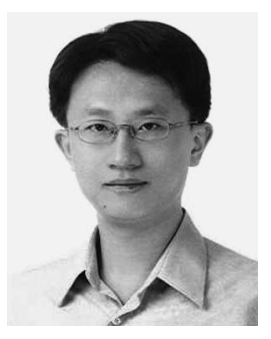

Hung-I Hsieh (S'00) received the B.S.E.E. degree from Chung Hua University, Hsinchu, Taiwan, R.O.C., in 2000 and the M.S.E.E. degree from National Chiao Tung University, Hsinchu, in 2002. He is currently working toward the Ph.D. degree in the Department of Electrical Engineering, National Taiwan University, Taipei, Taiwan.

His research interests include EMI in switching circuits, resonant power converters, modeling and control of switching power converters, digital PWM controller for power circuits, and power management IC design.

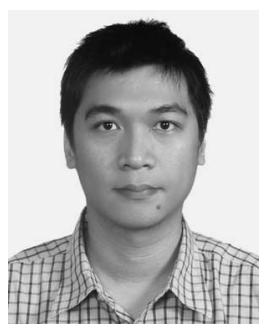

Jhong-Shu $\mathbf{L i}$ received the B.S.E.E. degree from the National Taiwan University of Science and Technology, Taipei, Taiwan, R.O.C., in 1999, the M.S.E.E. degree in 2003 from National Taiwan University, Taipei, where he is currently working toward the Ph.D. degree in the Department of Electrical Engineering.

His research interests include circuit control and modeling.

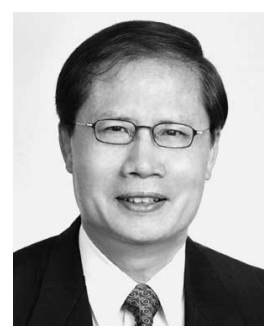

Dan Chen (S'72-M'75-SM'83-F'02) received the B.S.E.E. degree from National Chiao Tung University, Hsinchu, Taiwan, R.O.C., in 1969 and the $\mathrm{Ph} . \mathrm{D}$. degree in electrical engineering from Duke University, Durham, NC, in 1975.

From 1975 to 1979 , he was with the G.E. Corporate Research Center, Schecnectady, NY, where he worked on solid-state power applications including electronic ballasts for fluorescent lamps, electric cars, and switching power supply applications for computer and communication equipment. From 1979 to 2003, he was with the Department of Electrical Engineering, Virginia Polytechnic Institute and State University, Blacksburg, and was a Core Professor with the National Science Foundation's Center for Power Electronic Systems. Since August 2003, he has been a Professor with the Department of Electrical Engineering, National Taiwan University, Taipei, Taiwan. He has published more than 100 papers. He is the holder of seven U.S. patents, all in the field of power electronics. His recent research interests include piezoelectric transformers, high-power semiconductor device characterization, EMI in switching circuits, modern magnetic amplifiers for switching power supplies, and power management IC architecture and design.

Dr. Chen was the corecipient of the IEEE Aerospace Society Barry Carlton Award in 1973 and the Best Paper Award of the 1998 IEEE Power Electronics Society. He has published one IEEE-Press book and one tutorial article in the IEEE Spectrum magazine. 